\title{
Nanostructure Diffraction Analysis of a Copper/Single Walled Carbon Nanotube Nanocomposite Synthesized by Laser Surface Implanting
}

Jay F. Tuª, Nilesh Rajulea, Yi Liu' ${ }^{\text {ad }}$, and James Martin ${ }^{\mathrm{c}}$

aDepartment of Mechanical \& Aerospace Engineering, North Carolina State University, Raleigh, USA

${ }^{b}$ Department of Material Science and Engineering, North Carolina State University, Raleigh, USA 'Department of Chemistry, North Carolina State University, USA

dDepartment of Engineering Science, University West, Trollhättan, Sweden

Corresponding Author: Jay F. Tu, jftu@ncsu.edu

Short Title: TEM Indexing of a Copper/Carbon Nanotube Nanocomposite Classification numbers: $61,62,68,81$

\begin{abstract}
A new wet process has been developed to synthesize a Copper-Single Wall Carbon NanoTube (CU-SWCNT) metal nanocomposite by dispersing SWCNTs into molten copper, followed by rapid and non-equilibrium solidification to form the Cu-SWCNT nanocomposite such that dispersed SWCNTs could locked in positions without agglomerating into large clusters. However, the nanometer sizes of the SWCNT clusters make it extremely difficult to obtain TEM images with discernable SWCNT clusters in the copper matrix. In this paper, TEM images and their diffraction patterns for annealed pure copper, quenched pure copper (by the same synthesis process without introducing SWCNTs), and Cu-SWCNT nanocomposite are compared. It is concluded that TEM images with discernable SWCNT clusters are rare. Therefore, diffraction patterns are better tools to identify SWCNTs within the copper matrix. The indexed diffraction patterns confirm that the copper fcc lattice is preserved. However, the Cu-SWCNT nanocomposite samples also exhibit ordered diffuse scattering, consisting of at least two polyhedra of diffuse-scattering bounded by the $\{110\}^{*}$ and $\{200\}^{*}$ family of reciprocal lattice planes, respectively. In addition several samples exhibit super-lattice Bragg diffraction indicative expanded unit cells. It thus appears that the SWCNTs are incorporated into the $\mathrm{Cu}$ matrix with precise arrangements commensurate with specific $\mathrm{Cu}$ lattice planes.
\end{abstract}




\section{INTRODUCTION}

Carbon nanotubes (CNTs), since their discovery in 1991, have been the subject of intensive research. Due to their exceptional structural properties, CNTs are ideal reinforcing elements for composites in structural applications [1-3]. CNT-reinforced composite research first focused on polymer matrix composites because CNTs were envisioned as an alternative to carbon fibers. Subsequently interest grew in metal composites with CNTs as reinforcing elements. There are several challenges in the synthesis of CNT-metal composites for desirable properties. First, it is difficult to achieve uniform dispersion of CNTs as small clusters. This challenge is a result of CNTs' tremendous surface area, up to $200 \mathrm{~m}^{2} / \mathrm{g}$, for which van der Walls forces can lead to the formation of large clusters. The relative weakness of inter-CNT van der Walls interactions limits the strength of the metal composite at locations containing large CNT clusters, providing no improvement or even lower strength at those locations.

Summaries of the research effort on metal-CNT composite can be found in several reviews for which the greatest applied success in the use of CNT-reinforced aluminum for highfrequency MEMS resonators [4-6].

\subsection{REVIEW OF CONVENTIONAL DRY SYNTHESIS PROCESS OF CU-CNT COMPOSITES}

Synthesis of Cu-CNT composites is considered, in general, more difficult than Al-CNT composites because copper and carbon do not form intermetallic compounds as in the case for aluminum and carbon, which form aluminum carbide. Aluminum carbide, $\mathrm{Al}_{4} \mathrm{C}_{3}$, formation can help the Al-CNT formation by covalent bonding between Al and the CNT resulting in mechanical strengthening [7]. In addition, copper and carbon are mutually insoluble. As a result, the interfacial wettability of CNTs with the copper matrix becomes critical if a composite is to be formed.

The synthesis of $\mathrm{Cu}$-CNT composites has been reported by several research groups using powder metallurgy and ball milling with Ni-coated CNTs, combined with different sintering and cold forming processes [8-15]. Typical CNT volume fractions were between 5 and 10\%, 
with some as low as $1-2 \%$ or as high as $15 \%$. The hardness improvement was 20 to $110 \%$ (1.2 to 2.1 times) over that of pure copper.

A sandwich processing for fabricating Cu-CNT nanocomposites was presented in [16], in which pure thin copper foils of $10 \mu \mathrm{m}$ were acetone-sprayed with SWCNTs to achieve a $450 \mathrm{~nm}$ thickness of the SWCNT layer. With 19 SWCNT films sandwiched between 20 layers of pure copper foils, these laminates were then cold rolled and finally annealed at $1050^{\circ} \mathrm{C}$ for 10 hours in an argon atmosphere. Young's modulus was increased by $13 \% \pm 5 \%$.

The concept of Molecular Level Mixing was introduced in [17], using the spark plasma sintering process to fabricate Cu-MWCNT nanocomposites. Very elaborated processes were involved to produce CuO/CNTs, which were then converted to $\mathrm{Cu} / \mathrm{CNT}$ composite powder before spark plasma sintering to form Cu-MWCNT nanocomposite. It was observed that the yield strength of the composite could be improved up to $200 \%$ at $10 \%$ CNT volume fraction. Vickers hardness test of these composites showed up to $100 \%$ increase in hardness compared to that of pure copper was achieved, and wear resistance was increased up to three times that of pure copper [18-19] . However, the reported TEM images revealed that the CNT cluster sizes were still in the range of microns. Unless the CNT cluster sizes are in the range of nanometers, the goal of molecular level mixing cannot be claimed to have been achieved.

\subsection{A NEW WET SYNTHESIS OF CU-SWCNT NANOCOMPOSITES}

A new process, denoted as Laser Surface Implanting (LSI), for the synthesis of Cu-SWCNT nanocomposites by dispersing SWCNTs into molten copper, followed by rapid and nonequilibrium solidification to form Cu-SWCNT nanocomposites was presented in [20-21]. By this method, SWCNTs could be dispersed and locked in positions without agglomerating into large clusters. 

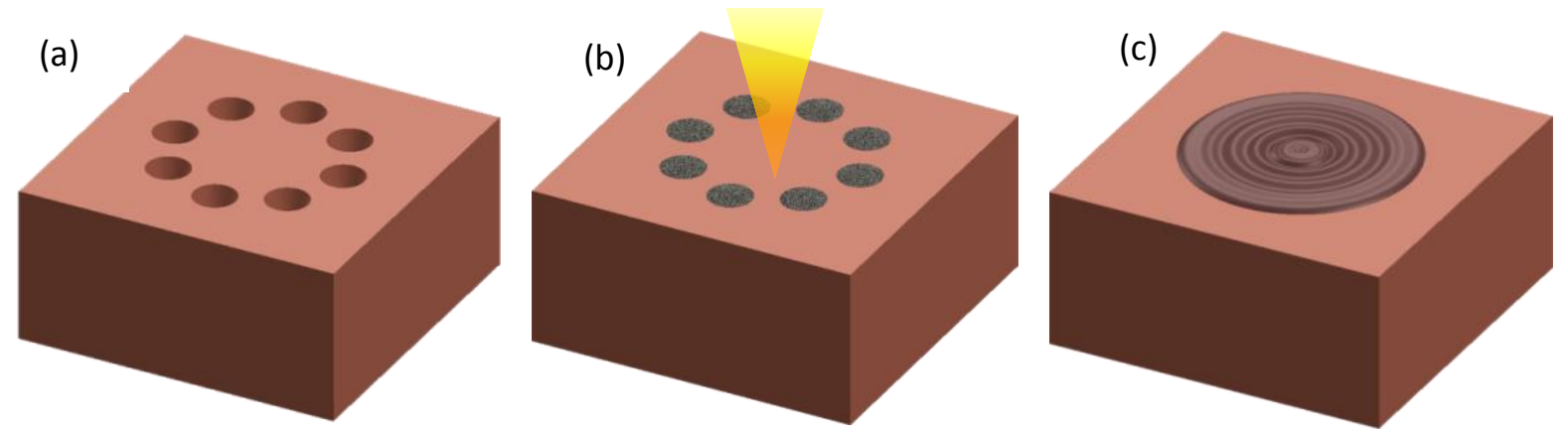

Figure (1): The reinforced copper substrate and the Laser Surface Implanting (LSI) process for synthesizing the SWCNT-Cu Nano-composite.

This wet process is very different from the typical dry powder metallurgy method. As shown in Figure (1), this novel synthesis process includes:

Step (1): A 300W single-mode fiber laser beam is focused to a $10 \mu \mathrm{m}$ spot size to drill a number of microholes (17 $\mu \mathrm{m}$ in diameter and depth) arranged around a circle of $70 \mu \mathrm{m}$ diameter (Figure (1a));

Step (2): The microholes are filled with SWCNTs suspended in solution (Figure (1b));

Step (3): The same laser is controlled to irradiate its beam at the center of the above micro-hole circle to create a well of molten copper, which advances radially outward to mix with the SWCNTs deposited inside the microholes and forms an Cu-SWCNT implant upon rapid, nonequilibrium solidification when the laser is turned off (Figure (1c)). Rapid and non-equilibrium solidification is achieved when the heat of the molten copper is rapidly conducted away into the copper substrate due to copper's excellent heat conductivity. The formation of the CuSWCNT composite is confirmed independently by energy-dispersive $\mathrm{X}$-ray spectroscopy mapping and spectroscopy measurements. The resulting material exhibited a hardness of over six times that of pure copper with only $0.23 \%$ volume fraction of SWCNTs. It is hypothesized that the SWCNT clusters deposited inside the microholes would remain dispersed when they are mixed with the molten copper because of the low volume fraction and the rapid solidification. Although the actual time scales of the solidification and agglomeration are unknown, it is likely the rapid solidification of the LSI process does not allow phase segregation, thus agglomeration of SWCNTs, to occur. Using TEM to verify the sizes of SWCNT clusters 
would confirm if this hypothesis is true or false. Technical details of this process are articulated in [20-21].

Because of the nano-dimensions of the SWCNT clusters, it is extremely difficult to obtain direct TEM images of SWCNT clusters in the copper matrix to confirm the nanostructure of the $\mathrm{Cu}$ SWCNT nanocomposite. In this paper, we present research methodologies and results on how to achieve useful TEM imaging as well as diffraction indexing of the Cu-SWCNT nanocomposite synthesized by the LSI.

\section{EXPERIMENTAL}

\subsection{Difficulties in TEM ChaRACtERIZATION OF THE CU-SWCNT NANOCOMPOSITE}

Because of the relative size of any nano-sized clusters of SWCNT inside the copper matrix with only $0.23 \%$ volume fraction only SWCNT clusters that occupy a space through the entire thickness are expected to be visible by TEM. This is because a sample with 40 to $50 \mathrm{~nm}$ thickness still contains

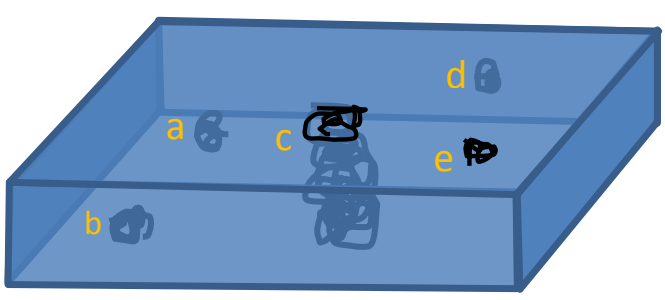

Figure (2): Different embedding of SWCNT clusters in a TEM sample. nearly 100 to 150 layers of copper atoms, which would dominate a TEM image obscuring the carbon atoms. Furthermore, were molecular-level isolation of SWCNT obtainable, the copper scattering would again be dominant, preventing direct visualization. The TEM sample usually cannot be made thinner due to the fixturing and transport of the sample to the TEM machine. This is schematically illustrated in Figure (2), in which cluster c would be visible by TEM if the TEM machine has sufficient sharpness and resolution However, SWCNT clusters such as those labeled as a, b, and d in Figure (2) would likely be invisible by TEM. Because of the dominant copper matrix, even cluster e that is exposed to the surface would not likely be discernable on a 
TEM image. In addition, the TEM machine must have very high resolution and the TEM sample must be meticulously prepared to achieve the required sharpness and focus of the image.

\subsection{TEM Sample Preparation for JEOL 2010F STEM}

TEM imaging was conducted on JEOL 2010F STEM. Sample preparation was carried out to thin the samples to a thickness less than $40 \mathrm{~nm}$ with very smooth surface finish. TEM samples were prepared on FEI Quanta 3D Dual Beam SEM/FIB operating at 30 kV using "Lift-Out" technique. The FIB sample preparation typically takes 3-5 hours due to the thinness requirement, while TEM inspection about 7 to 10 hours, and post-analysis about 6 to 9 hours for one sample. Therefore, it is cost prohibitive to produce many samples to cover the entire Cu-SWCNT implant.

Below is a summary of the sample preparation steps.

An FEI Quanta 3D Dual Beam Scanning Electron Microscope (SEM) with focused ion beam (FIB) was used to prepare the TEM specimens. The lift-out technique was used in TEM specimen preparation. Firstly, a platinum bar with a dimension about 10 um $\times 2$ um $\times 2$ um was deposited using the ion beam and Pt injector in order to protect the area of interests. Then, a volume with the dimension of 15 um $\times 8$ um $\times 8$ um on both sides of the platinum bar were milled away by the FIB at relatively higher probe current, typically $15 \mathrm{nA}$. The thin foil was picked up by the Omniprobe which is a sharp tip made by tungsten and then welded onto a Cu half-ring grid by depositing a small square of Pt for further thinning to yield the TEM sample. The microscope was operated at $30 \mathrm{kV}$ on both SEM and FIB sides initially. At the final polishing stage of the FIB, the voltage was lowered to $5 \mathrm{kV}$ and probe current about 10-30 pA to minumize the damage introduce by the ion beam. 


\section{RESULTS}

\subsection{TEM Images by JEOL 2010F STEM}

Figure (3) presents two TEM images, in which distinctive SWCNT clusters embedded in the copper matrix can be discerned. To authors' best knowledge, this is the first time a TEM image is produced to show such nano-sized cluster of SWCNTs in a CU-SWCNT nanocomposite. These SWCNT clusters, curved in shape, range from 5 to $30 \mathrm{~nm}$ in size. In addition, there exist many Moiré fringes in the vicinity of carbon nanotubes, illustrated by green arrows in the TEM images. Moiré fringes likely are generated due to the overlapping of more than one thin crystal


Figure (3): TEM images obtained by JEOL 2010F STEM. The Moire Fringes are indicated by green arrows, while discernable SWCNT clusters are indicated by the yellow arrow.

with similar periodic arrangements of atoms. Though not conclusive, the abundance of Moiré fringes may be indicative of the existence of SWCNTs in the copper matrix. During the solidification of the copper, due to the presence of SWCNT clusters, a single crystal could grow until its solidification is interrupted by SWCNTs which introduce defects to the crystal lattice. The solidification of copper then continues with a slight adjustment to the orientation of the crystal lattice. 
The very small sizes of the observed SWCNT clusters, 5 to $30 \mathrm{~nm}$, indicate that the SWCNTs are well dispersed during the LSI process despite the non-wetting nature between SWCNTS and molten copper. This finding appears to support the hypothesis stated in Section 1.2 regarding the solidification time of the LSI process could be short enough to prevent SWCNT agglomeration. However, not all the images obtained from this sample contain discernable

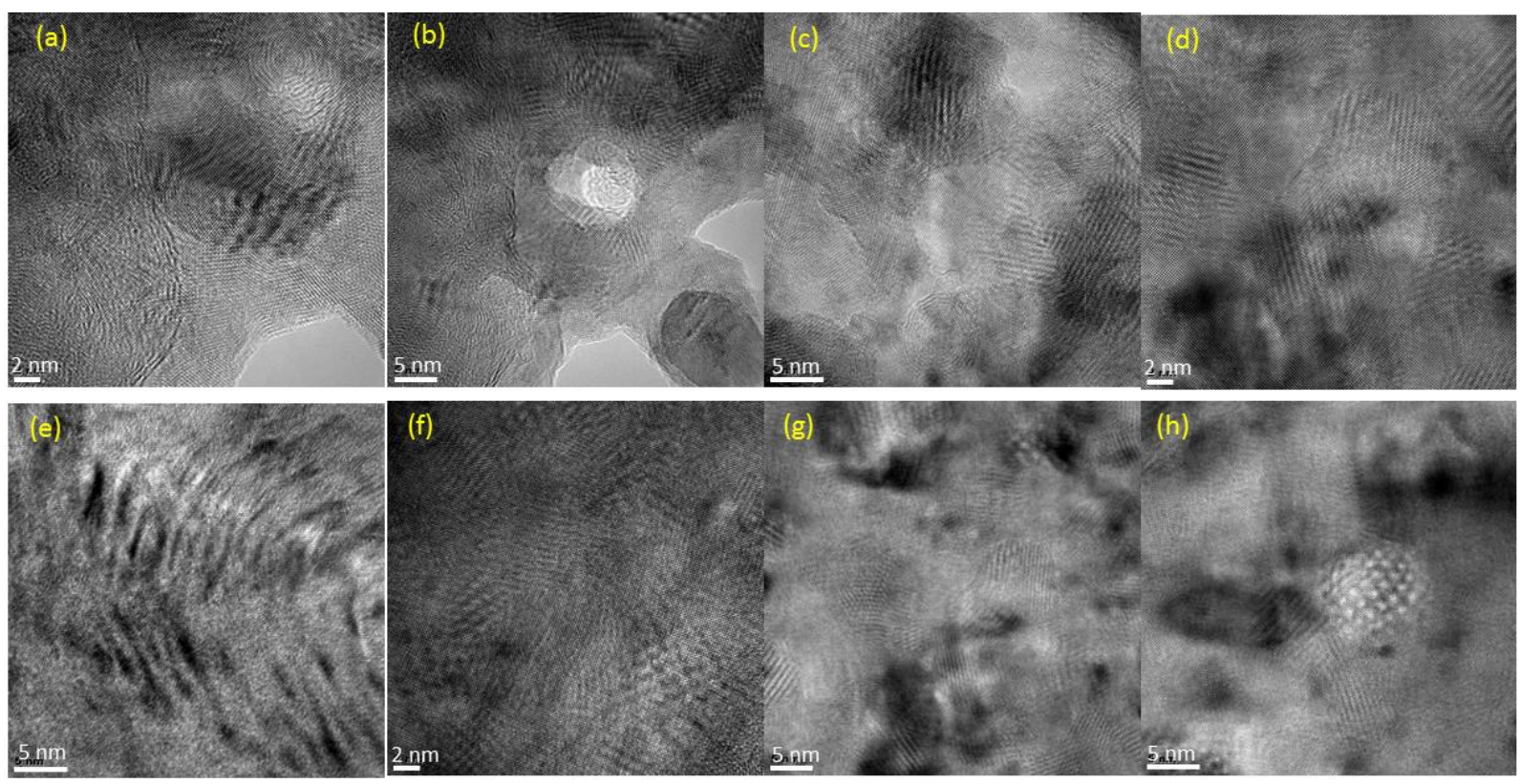

Figure (4): Direct TEM images of Cu-SWCNT.

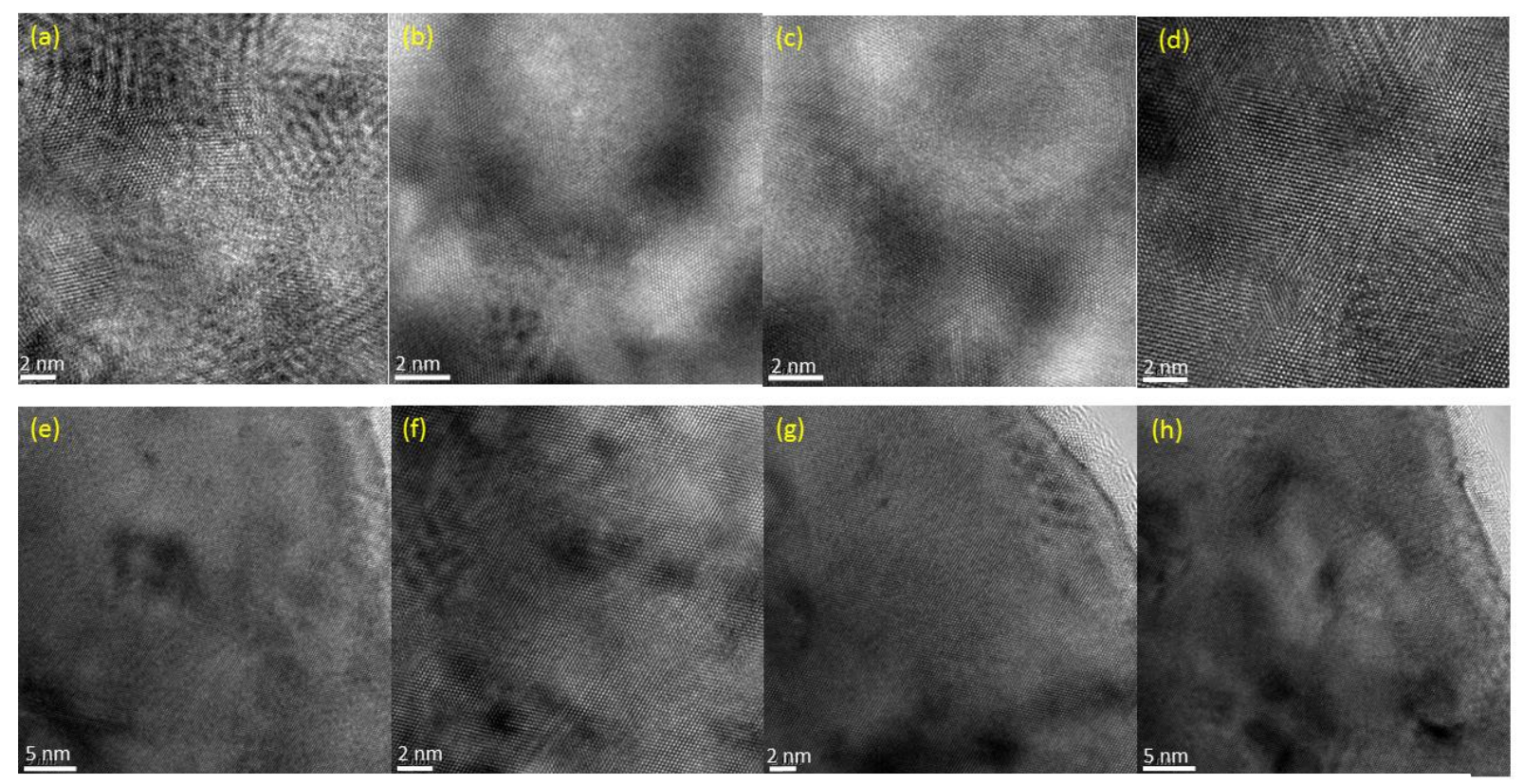

Figure (5): Direct TEM images of copper with and without laser melting and rapid solidification. 
SWCNT clusters. Several images are reproduced in Figure (4). Among them, significant evidence of SWCNT clusters could only be found in images a, b, c, and h, though diverse Morié fringes are observed in images $d$ to $g$. We also obtained TEM images of pure copper for comparison. The ordered pattern in the middle of Figure (4h) likely is the "cross-section" of an SWCNT bundle, similar to the TEM image published in [22]. In Figure (4h), the SWCNTs are oriented with their axes in parallel to the beam direction of the TEM machine. The SWCNTs are close-packed with a rectangular arrangement, compared with the triangular arrangement in [22], while the diameter of the tube and the spacing between tubes are similar, with approximately four tubes closely packed in a line of $5 \mathrm{~nm}$.

Eight TEM images of pure copper are reproduced in Figure (5). Images a to $c$ in Figure (5) were obtained from a pure copper sample that was not subjected to the LSI process (annealed copper). Images $d$ to $h$ were obtained from a pure copper sample which was processed by LSI without introducing SWCNTs into the sample, i.e. subject to the same melting and subsequent rapid solidification (quenched copper) as the CU-SWCNT composite. We prepared these two pure copper samples as controls to determine the extent to which the melting and rapid solidification of pure copper affects the characteristics of the TEM images. In images e, $g$, and $h$ of Figure (5), the upper right hand corner is vacuum. The images in Figure (5) in general contain only a few or no Moiré fringes, unlike those in Figure (4). However, it would be difficult to confirm the existence of SWCNTs based on the TEM images unless distinctive SWCNT clusters could be found.

The existence of some Moiré fringes in the control sample of quenched copper suggests that the LSI processing itself may be responsible for a certain amount of the structural disorder resulting in the Moiré fringes and diffuse scattering. This is not totally unexpected because the rapid solidification of the LSI process would introduce smaller grains during solidification.

\subsection{Diffraction Pattern via FFT}

Converting a TEM image into a diffraction pattern via the fast Fourier transform (FFT) method is an effective method for crystallographic analysis. It is generally advised not to take any TEM image of a crystal without a corresponding diffraction pattern. The diffraction patterns in this 
paper were obtained using the software "Gatan Digital Micrograph" installed on JEOL 2010F to control its digital camera system, calibrated by authorized JEOL engineers. The area selected for FFT was usually about $20 \mathrm{~nm} \times 20 \mathrm{~nm}$. The unit of the TEM image and that of its diffraction pattern are reciprocal. The TEM image is in the real space, with a unit such as $\mathrm{nm}$, while the diffraction pattern is in the reciprocal space, with a unit such as $1 / \mathrm{nm}$.

Figure (6) shows the diffraction pattern of the TEM image of Figure (5-a), a pure copper sample without melting and rapid solidification (annealed copper).

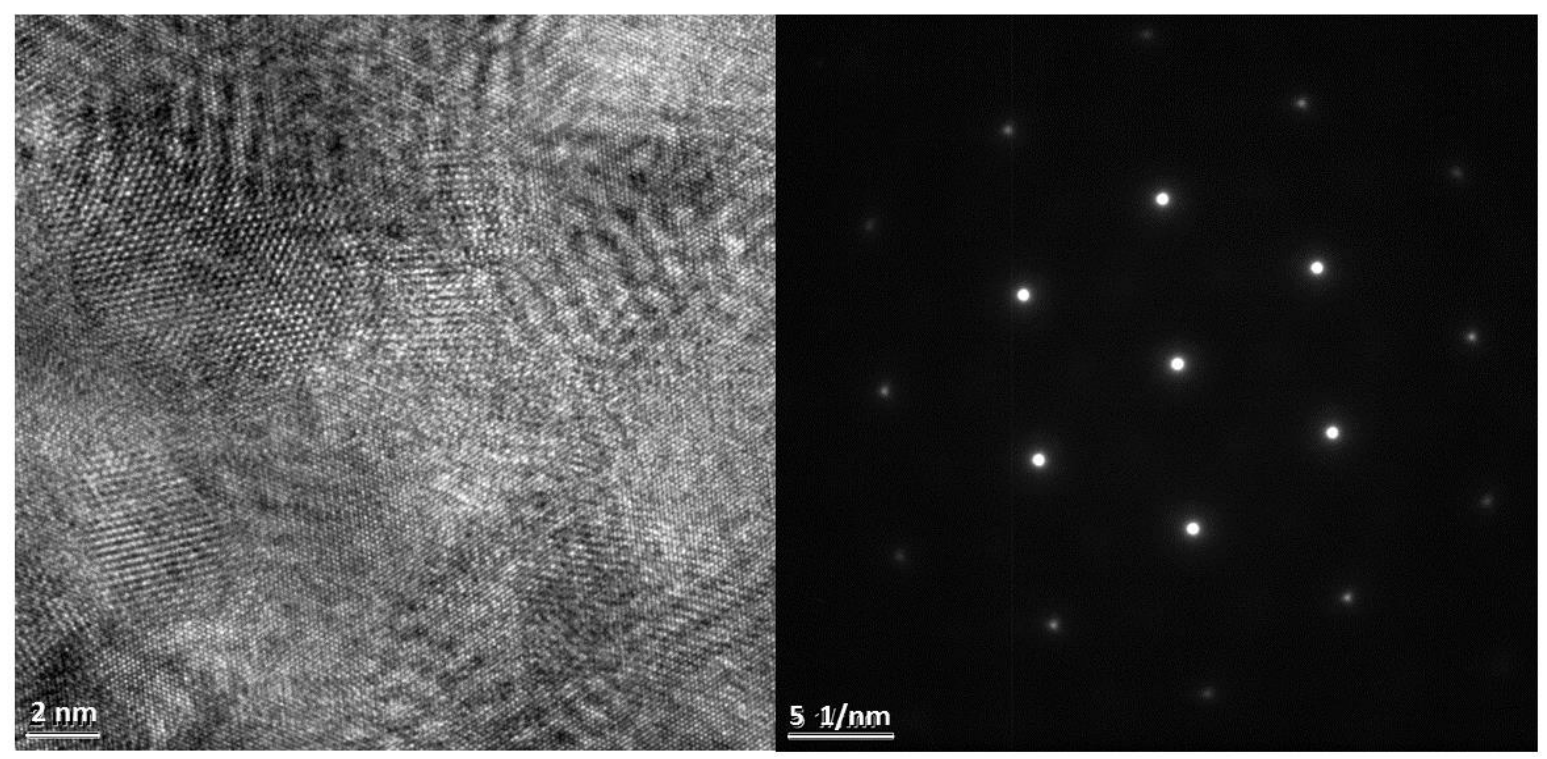

Figure (6): a TEM image of annealed pure copper and its diffraction pattern.

Figure (7) presents the diffraction pattern of the TEM image of Figure (5-e), a sample which was processed by the same LSI process, but without introducing SWNTs into the microholes (quenched copper). Both of these diffraction patterns exhibit distinctive $f c c$ diffraction patterns, [111] and [110] oriented, respectively, with clean backgrounds. Both diffraction patterns indicate perfect single crystals. 


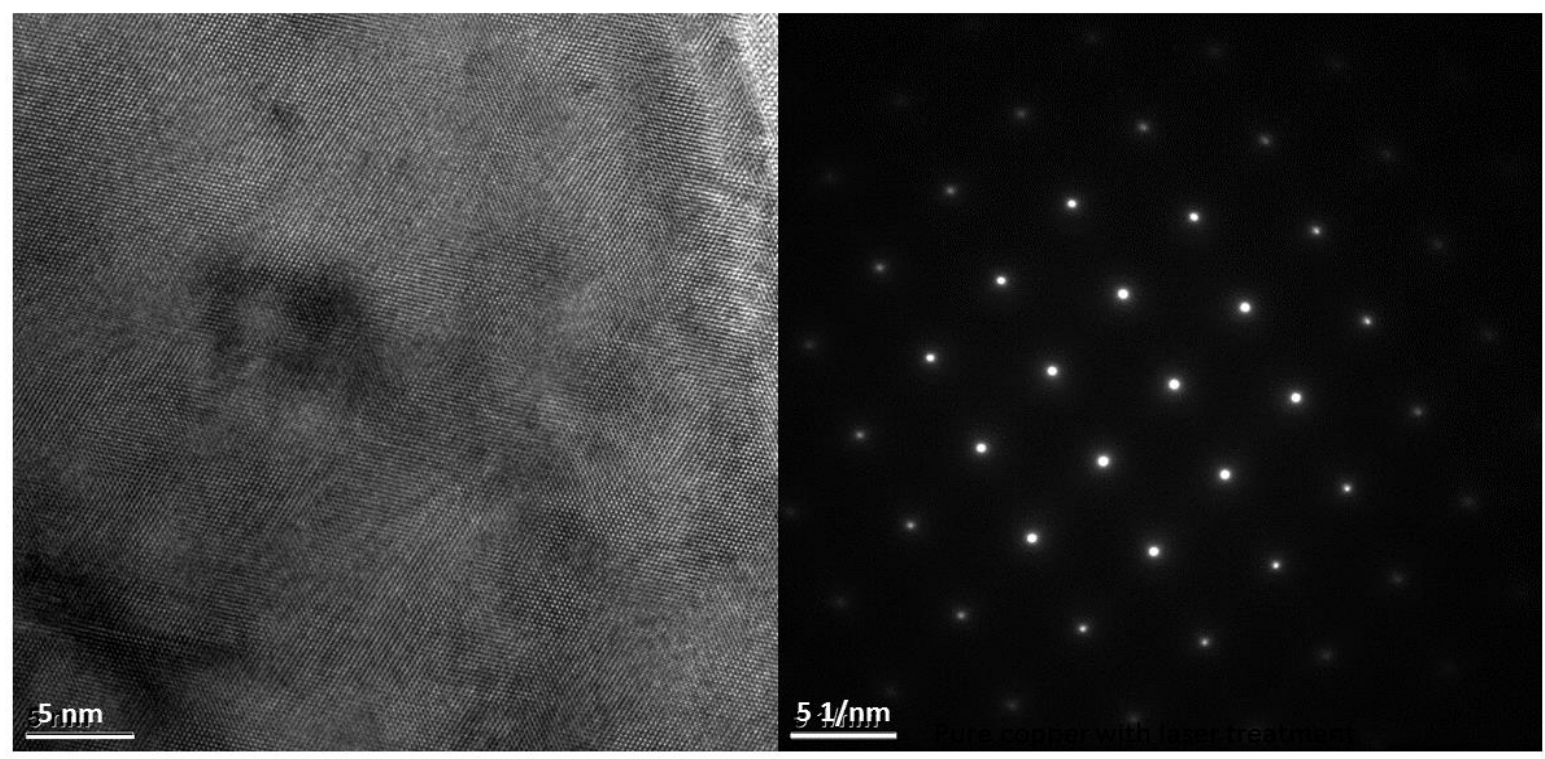

Figure (7): a TEM image of quenched pure copper and its diffraction pattern

By contrast, Figure (8) shows the FFT of the TEM image of Figure (4-c), a Cu-SWCNT sample. The diffraction pattern of the Cu-SWCNT is distinctively different from those of pure copper, with or without the LSI processing.

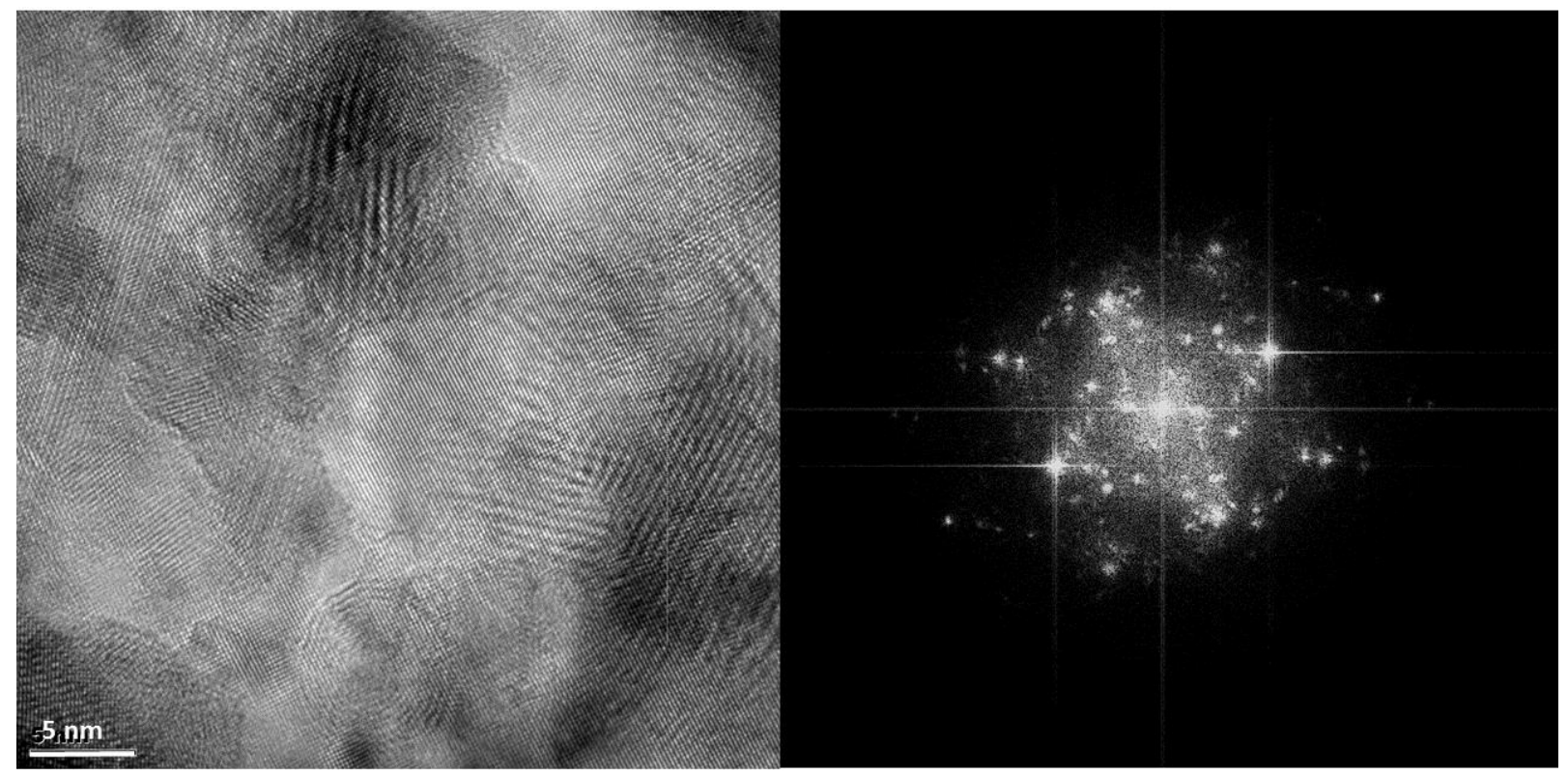

Figure (8): The TEM image of Cu-SWCNT in Figure (4-c) and its diffraction pattern. 


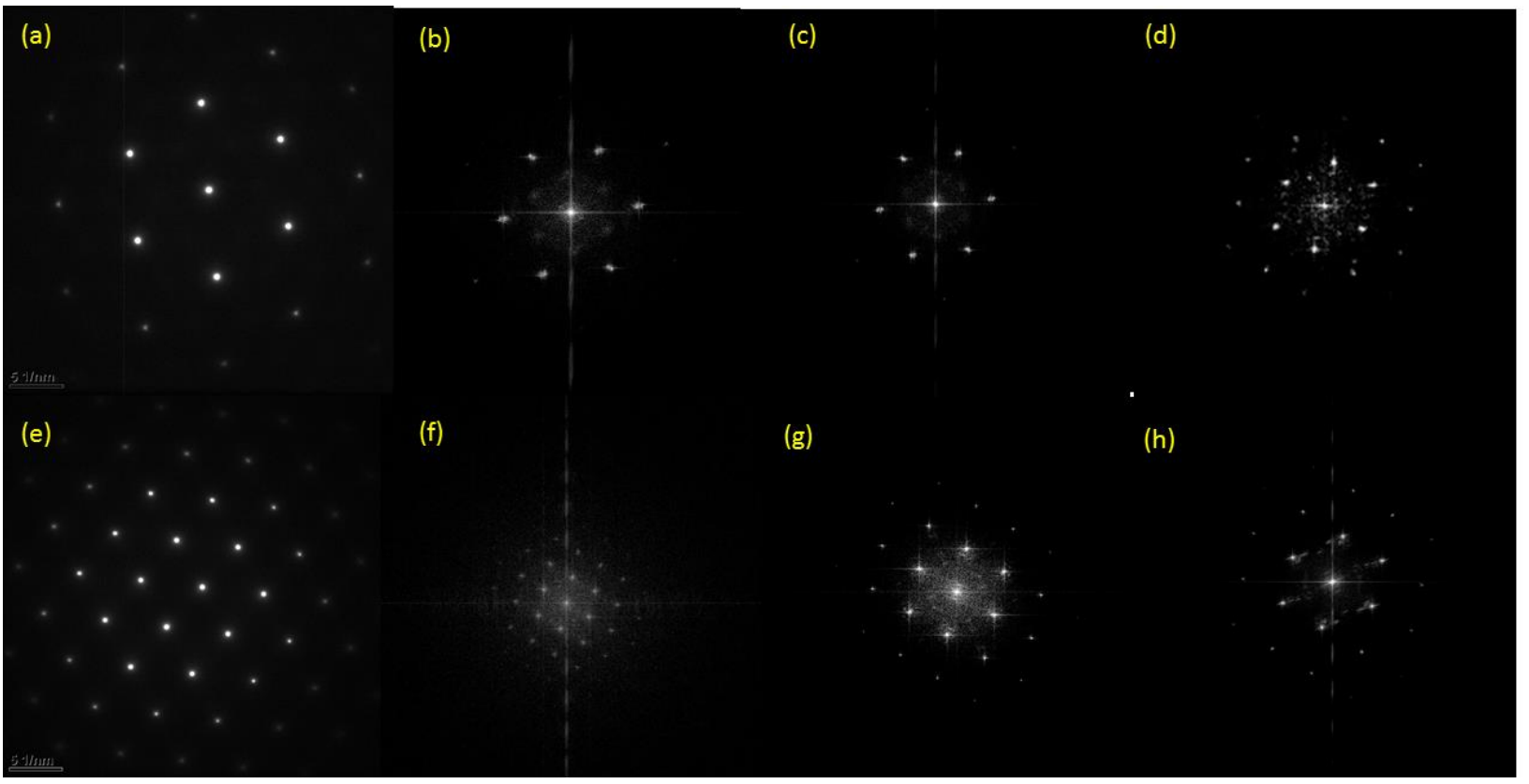

Figure (9): Diffraction patterns of all TEM images of Figure (5) for pure copper

(a)

(b)

(c)

(d)

(e)

(f)

(g)

(h)

Figure (10): Diffraction patterns of all TEM images of Figure (4) for Cu-SWCNT nanocomposite.

FFT analysis was applied to all TEM images in Figure (4) and (5), yielding Figures (10) and (9), respectively. Notably, the primary diffraction spots of the Cu-SWCNT samples directly index to the fcc-Cu lattice. In addition, these exhibit a substantially increased amount of diffuse scattering and super-lattice diffraction spots. The diffuse scattering and super-lattice structure 
indicates with certainty that the Cu-SWCNT samples and the pure copper samples (with or without LSI processing) have different crystalline organization.

\subsection{Indexing for the Diffraction Patterns of Pure Copper}

Diffraction indexing was carried to identify the crystalline structure of the samples. Not every image in Figures (4) and (5) is identically aligned; the orientation is generally unknown until the diffraction pattern is obtained and indexed. The TEM diffraction images of Figure (6) (annealed) and (7) (quenched) are indexed in Figure 11, and are found to be [111] and [011] oriented, respectively. Although the orientation is distinct there is no discernable difference between the annealed pure copper sample and the LSI quenched pure copper sample. For the [111] oriented diffraction patter of Figure (11), left, the ring radius of each family of planes, $\{220\},\{422\}$, and $\{440\}$, is labeled as $L_{1}, L_{2}$, and $L_{3}$, respectively. Similarly, for the [011] oriented diffraction pattern of Figure (12), right, the corresponding ring radius for each family of $\{11 \overline{1}\},\{200\}$, and $\{02 \overline{2}\}$, is labeled as $M_{1}$, $M_{2}$, and $M_{3}$, respectively.

Because copper exhibits a fcc structure, $a=3.61 \AA$, the spacing $d_{h k l}$ between adjacent $(h k l)$ lattice planes is,

$$
d_{h k l}=\frac{a}{\sqrt{h^{2}+k^{2}+l^{2}}}
$$

The corresponding reciprocal lattice vectors, $r_{h k l}$, observed in the diffraction pattern can be described as

$$
r_{h k l}=\frac{L \lambda}{d_{h k l}}=\frac{L \lambda \sqrt{h^{2}+k^{2}+l^{2}}}{a}
$$

Where $L$ is the camera length.

Based on Equations (1) and (2), if the diffraction patterns of Figure (11) are from copper samples, the ratios of the lattice vectors shown in Figure (11) must agree with the following relationships:

$$
\begin{gathered}
\frac{L_{2}}{L_{1}}=\frac{\sqrt{4^{2}+2^{2}+2^{2}}}{\sqrt{2^{2}+2^{2}+0^{2}}}=\frac{\sqrt{3}}{1} ; \frac{L_{3}}{L_{1}}=\frac{\sqrt{4^{2}+4^{2}+0^{2}}}{\sqrt{2^{2}+2^{2}+0^{2}}}=\frac{2}{1} \\
\frac{M_{2}}{M_{1}}=\frac{\sqrt{2^{2}+0^{2}+0^{2}}}{\sqrt{1^{2}+1^{2}+1^{2}}}=\frac{2}{\sqrt{3}} ; \frac{M_{3}}{M_{1}}=\frac{\sqrt{0^{2}+2^{2}+2^{2}}}{\sqrt{1^{2}+1^{2}+1^{2}}}=\frac{\sqrt{8}}{\sqrt{3}}
\end{gathered}
$$


The above ratios are confirmed for both copper samples.
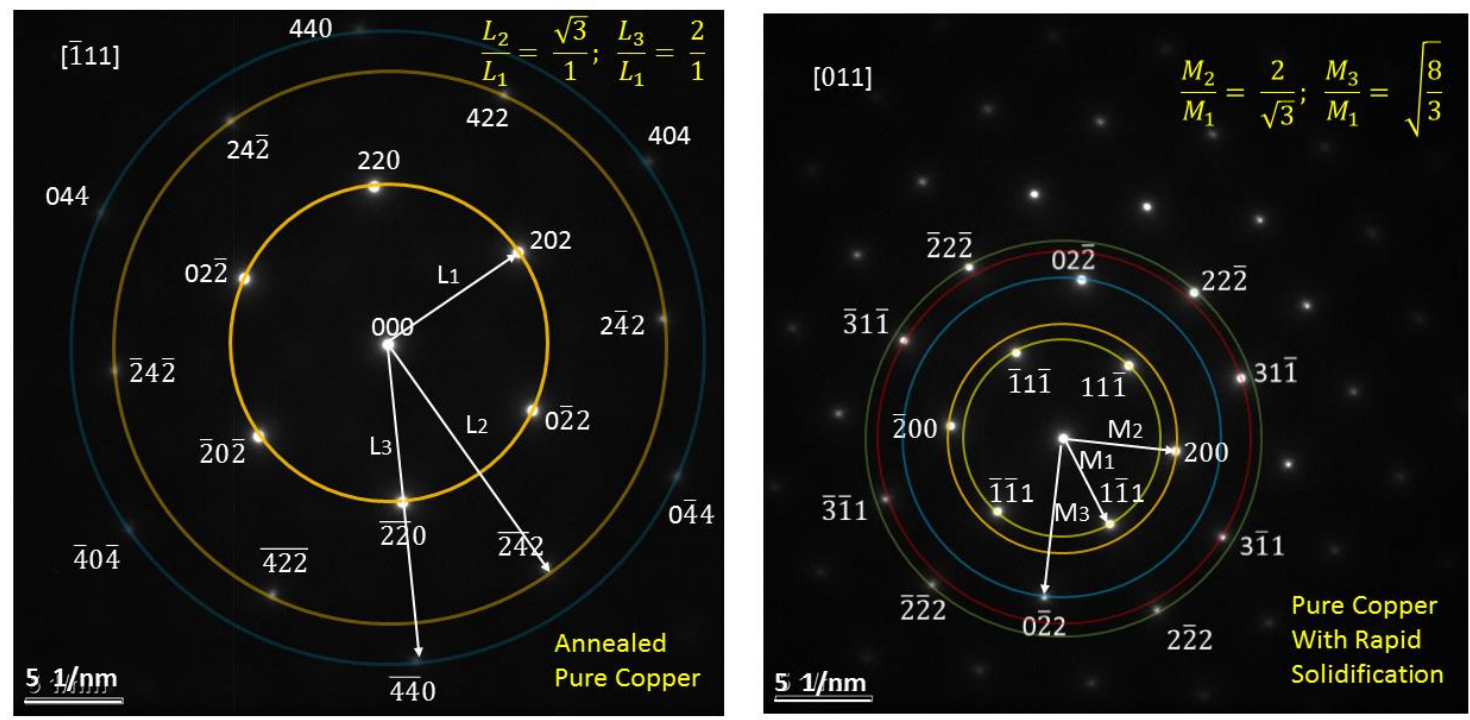

Figure (11): Indexed diffraction patterns of pure copper, with and without laser melting.

\subsection{Indexing for the Diffraction Pattern of the Cu-SWCNT Composite}

The same indexing strategy was applied to the diffraction patterns of Figure (10). Image (10-e) exhibits an [110] orientation, while images (10-a-d and $f-h)$ are [010] oriented. Detailed indexing is given in Figures (12a) and (12b) for images (10-a) and (10-e), respectively. The sample corresponding to image of $(12-b)$ exhibits only lower-angle diffraction peaks $<(111)$ indicating that it is less crystalline that all the other images which exhibit sharp $f c c$ diffraction out to (420). Note that this low-angle diffraction could also happen if the crystal is not oriented well along the electron beam, or simply due to the thickness of the sample at that area is relative thick.

Unlike the diffraction patterns for the pure copper samples, Figure (9), the diffraction patterns for the Cu-SWCNT samples, Figure (10), exhibit extensive diffuse scattering, indicative of disorder. Note that even though each tube of SWCNTs has very ordered structure of carbon atoms, and the diffraction pattern of one single SWCNT would display an ordered pattern as those presented in [23], this is not the case when many SWCNT tubes are tangled together to form a bundle of SWNTs. Because different tubes are oriented differently within the bundle, the carbon atoms, as a whole, are no longer orderly arranged. Therefore, in Figures (10) and (12), the SWCNT atoms would display diffraction patterns as seemly random bright clouds, diffusely scattered. 

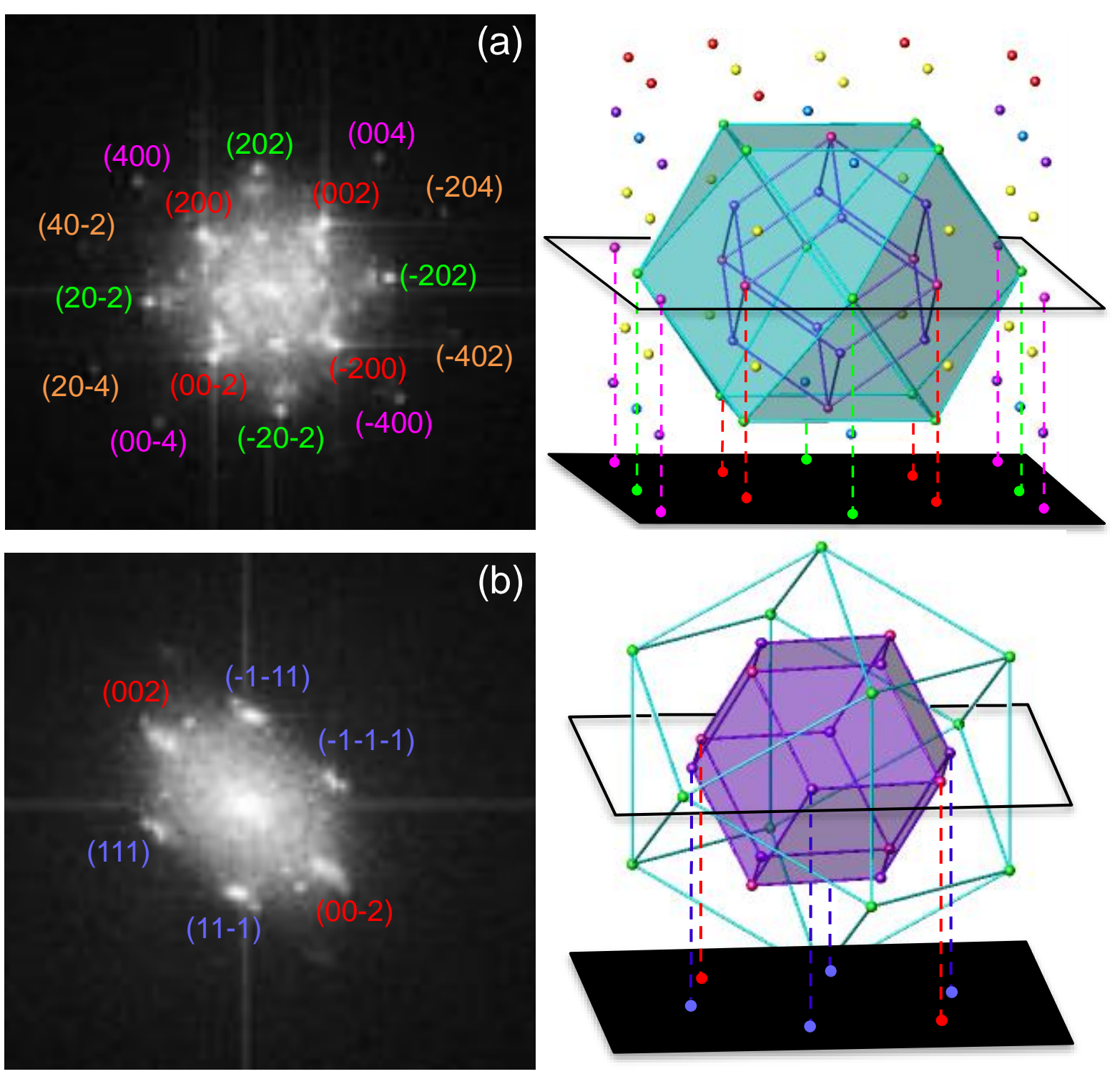

Figure (12): Indexed diffraction images and schematic of the Ewald sphere and its projection corresponding to the Cu-SWCNT TEM diffraction images from (a) Figure (10-a) and (b) Figure (10-e).

\section{Discussion}

\subsection{Patterns of Diffuse Scattering}

However, although the SWCNT atoms within the copper matrix display diffraction patterns as seemly random bright clouds, diffusely scattered, this diffuse scattering is clearly ordered, with two primary features. All of the [010] oriented samples exhibit a larger square of diffuse with reciprocal space [200]* edges and (220)* vertices as well as a smaller square of diffuse with reciprocal space [110]* edges and 
(200)* vertices. The distorted hexagon of diffuse observed in [110] oriented image of Figure (12b) is equivalent, but in a different orientation, to that of the smaller square of the [010] oriented images, indicating that these represent a polyhedron of diffuse, bounded by (200)* and (111)* Bragg reflections. The faces of the polyhedron are the $\{110\}^{*}$ reciprocal lattice planes, thus we will refer to this as the $\{110\}$-diffuse. This is represented as the purple polyhedron in the schematics of the Ewald sphere and its projections in Figure (12). We do not have a [110] oriented image that exhibits the higher order diffuse, but we presume it to also consist of a polyhedral volume of diffuse, blue polyhedron in Figure (12), for which $\{200\}^{*}$ reciprocal lattice planes are the polyhedral faces, thus we will refer to this as the $\{200\}$ diffuse.

\subsection{Indication of Super-Structure}

These ordered diffuse patterns clearly indicate that the SWCNTs incorporation into the Cu-lattice is not completely random. A random orientation of many nanotubes would result in rings of diffuse, which are clearly not observed. Instead, the ordered diffuse scattering is reminiscent of that observed in [24] for the plastic crystal structure of $\mathrm{CBr}_{4}$. There an overall $\mathrm{fcc}$ lattice of $\mathrm{CBr}_{4}$ molecules dictates the primary Bragg diffraction, but displacive distortions along the molecular nearest-neighbor $1 / 2<110>$ give rise to distinct sheets of diffuse. Here, the reasonably sharply defined polyhedral faces of diffuse likely indicate that the SWCNTs are directionally aligned within the $\{110\}$ and $\{200\}$ families of planes of the $f c c \mathrm{Cu}$ lattice. Unlike the plastic crystal of $\mathrm{CB}_{4}$, for which narrow sheets of diffuse are observed, a variable periodicity (e.g. nanotubes in random layers) could be the cause of faceted volume of diffuse, as opposed to sheets or rods of diffuse.

In addition to the clearly ordered diffuse scattering in the diffraction images of Figure (10), weak Bragg diffraction spots are observed between the strong $\mathrm{Cu}$ sub-lattice diffraction spots, which appear to be oriented commensurate with the copper lattice. The appearance of this super-lattice diffraction seems to be inversely correlated with the intensity of the diffuse scattering. The inverse correlation between the diffuse and super-lattice scattering suggests both originate from common structural components, just with differing extents/length scales of ordering. Certain of the additional spots can potentially be accounted for by crystal twinning (e.g. the apparent diamond of spots in image (10-b) and shifted pseudo hexagon of (10-e)). However, numerous spots are observed at smaller diffraction angles than the lowest allowed $f c c$ diffraction spots. In images (10-a) and (10-b), the extinct 110 reflections seem to be present, indicative of at least a doubled Cu-lattice. An even finer grid of super-lattice spots is 
observed in the other images. An expanded image of (10-f) is given in Figure (13), for which green circles mask the Cu-fcc lattice spots. Here super-lattice diffraction spots, indicate an apparent 7-fold super-structure along $\mathbf{a}$ and 5 -fold super-structure along $\mathbf{b}$ lattice vectors, indicated by arrows in Figure (13).

Careful examination of the pure copper samples of Figure (9) shows a muchreduced extent of diffuse scattering in some of the samples. Interestingly, the diffuse scattering is observed in the same samples for which the corresponding TEM images of LSI processed samples without SWCNTs also demonstrate some Moiré fringes. The LSI processing and/or the introduction of impurities may responsible for a certain amount of the diffuse. However, none of these quenched copper samples exhibit the clear super-lattice diffraction spots.

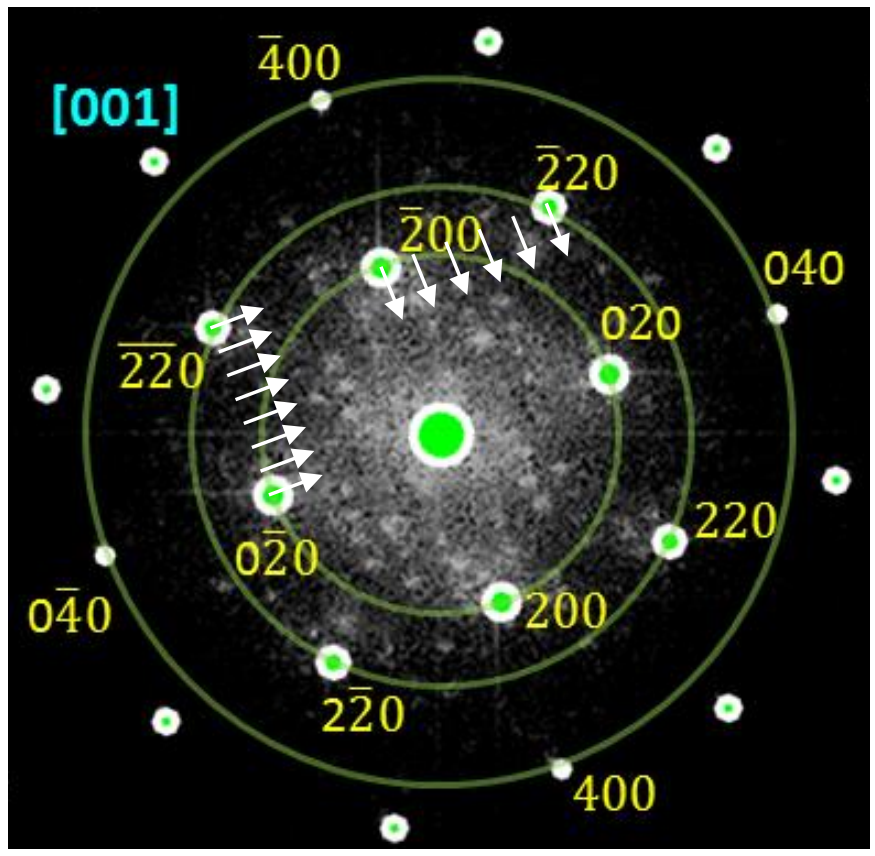

Figure (13): Expanded view of diffraction image 11f. Green circles mask $\mathrm{Cu}-\mathrm{fcc}$ lattice spots. White arrows describe the $7 \times 5$ super-structure.

The apparent collapse of some of the

diffuse scattering intensity into super-lattice diffraction spots suggests that under certain conditions, the SWCNTs become significantly ordered into the Cu-fcc sub-lattice, possibly organized in a similar fashion to cubic lyotropic liquid crystals. The difference between images (10-a) and (10-e) exhibiting the most diffuse, and (10-f) and (10-h) the most distinct super-structure diffraction spots, likely originates from a different concentration of SWCNTs, or different cooling profiles for the distinct samples.

\subsection{Distribution of SWCNTs Inside the Copper Matrix}

From Figure 4(a)(b)(c), it appears that the CNTs are easily laying on $\{001\}$ planes of Cu, i.e. CNTs parallel to $\{001\}$ planes. Carbon has smaller atom radius, could go into the interstitial locations in FCC structure, as how carbon go into Austenite in steel. However, to confirm this observation, many samples and more TEM images are needed, which could be an important topic for future studies. 


\section{CONCLUSION}

A new wet process has been developed to synthesize a Copper-Single Wall Carbon NanoTube (CU-SWCNT) metal nanocomposite by overcoming serious issues regarding wettability and fine dispersion of SWCNTS within the copper matrix. We have overcome difficulties and obtained TEM images showing SWCNT clusters in the nanometer sizes within the copper matrix. We presented the meticulous sample preparation method, needed for producing useful TEM images. TEM images for annealed pure copper, quenched pure copper (by the LSI process without introducing SWCNTS), and Cu-SWCNT nanocomposite are compared. Their corresponding diffraction patterns were then derived using FFT. Direct visualization of the SWCNTS by TEM is very rare. By contrast, diffraction patterns clearly identify novel structure of the Cu-SWCNT composites. The indexed diffraction patterns indicate that to overall $\mathrm{Cu}-\mathrm{fcc}$ lattice is maintained. However, remarkably ordered diffuse scattering, and/or the presence of super-lattice diffraction spots indicate that the SWCNT are directionally aligned within the $\{110\}$ and $\{200\}$ families of planes. The extent of alignment is likely dependent on concentration and annealing conditions.

\section{Acknowledgement}

The authors would like to acknowledge NSF DMII Grant \#0550734 as well Engineering On Line, Department of Mechanical and Aerospace Engineering of NCSU, the CHA!N group, and the Knowledge Foundation of Sweden for their supports and assistance. This work was performed in part at the Analytical Instrumentation Facility (AIF) at North Carolina State University, which is supported by the State of North Carolina and the National Science Foundation (award number ECCS-1542015). The AIF is a member of the North Carolina Research Triangle Nanotechnology Network (RTNN), a site in the National Nanotechnology Coordinated Infrastructure (NNCl).

\section{References}

1. Iijima, S., 1991, "Helical microtubules of graphitic carbon", Nature, vol. 354, no. 6348, pp. 56 to 58 .

2. Ajayan, P.M. and Zhou, O.Z., 2001, “Applications of Carbon Nanotubes”, in Dresselhaus, M.S., Dresselhaus, G. and Avouris, P. (Eds.),Carbon Nanotubes, Topics in Applied Physics, Springer Berlin Heidelberg, pp. 391 to 425. 
3. Endo, M., Strano, M.S. and Ajayan, P.M., 2007, "Potential Applications of Carbon Nanotubes", in Jorio, A., Dresselhaus, G. and Dresselhaus, M.S. (Eds.),Carbon Nanotubes, Topics in Applied Physics, Springer Berlin Heidelberg, pp. 13 to 62.

4. Curtin, W.A. and Sheldon, B.W., 2004, "CNT-reinforced ceramics and metals", Materials Today, vol. 7, no. 11, pp. 44 to 49.

5. Carreño-Morelli, E., 2009, “Carbon Nanotube-Metal Matrix Composites", Dekker Encyclopedia of Nanoscience and Nanotechnology, Second Edition, Taylor \& Francis, pp. 611 to 619 .

6. Agarwal, A., Bakshi, S.R. and Lahiri, D., 2011, Carbon Nanotubes: Reinforced Metal Matrix Composites, CRC Press.

7. Esawi, A.M.K., Morsi, K., Sayed, A., Gawad, A.A. and Borah, P., 2009, "Fabrication and properties of dispersed carbon nanotube-aluminum composites", Materials Science and Engineering: $A$, vol. 508, no. 1-2, pp. 167 to 173.

8. Dong, S. and Zhang, X., 1999, "Mechanical Properties of Cu-based Composites Reinforced by Carbon Nanotubes", Transactions of Nonferrous Metals Society of China, vol. 9, no. 3, pp. 457 to 461.

9. Dong, S.R., Tu, J.P. and Zhang, X.B., 2001, "An investigation of the sliding wear behavior of Cu-matrix composite reinforced by carbon nanotubes", Materials Science and Engineering: A, vol. 313 , no. $1-2$, pp. 83 to 87.

10. Chen, X., Li, W., Chen, C., Xu, L., Yang, Z. and Jing, H., 2005, “Preparation and properties of Cu matrix composite reinforced by carbon nanotubes", Transactions of Nonferrous Metals Society of China, vol. 15, no. 2, pp. 314 to 318.

11. Tu, J.P., Yang, Y.Z., Wang, L.Y., Ma, X.C. and Zhang, X.B., 2001, "Tribological properties of carbon-nanotube-reinforced copper composites", Tribology Letters, vol. 10, no. 4, pp. 225 to 228.

12. Kim, K.T., Lee, K.H., Cha, S.I., Mo, C.-B. and Hong, S.H., 2004, “Characterization of Carbon Nanotubes/Cu Nanocomposites Processed by Using Nano-sized Cu Powders", MRS Proceedings, vol. 821 , pp. 3 to 25 
13. Guiderdoni, C., Estournès, C., Peigney, A., Weibel, A., Turq, V. and Laurent, C., 2011, "The preparation of double-walled carbon nanotube/Cu composites by spark plasma sintering, and their hardness and friction properties", Carbon, vol. 49, no. 13, pp. 4535 to 4543.

14. Pham, Q., Jeong, Y.G., Hong, S.H. and Kim, H.S., 2006, "Equal Channel Angular Pressing of Carbon Nanotube Reinforced Metal Matrix Nanocomposites", Key Engineering Materials, vol. 326-328, pp. 325 to 328.

15. Quang, P., Jeong, Y.G., Yoon, S.C., Hong, S.H. and Kim, H.S., 2007, “Consolidation of 1vol.\% carbon nanotube reinforced metal matrix nanocomposites via equal channel angular pressing", Journal of Materials Processing Technology, vol. 187-188, pp. 318 to 320.

16. Li, Q., Viereckl, A., Rottmair, C.A. and Singer, R.F., 2009, "Improved processing of carbon nanotube/magnesium alloy composites", Composites Science and Technology, vol. 69, no. 78, pp. 1193 to 1199.

17. Cha, S.I., Kim, K.T., Arshad, S.N., Mo, C.B. and Hong, S.H., 2005, "Extraordinary Strengthening Effect of Carbon Nanotubes in Metal-Matrix Nanocomposites Processed by Molecular-Level Mixing", Advanced Materials, vol. 17, no. 11, pp. 1377 to 1381.

18. Kim, K.T., Cha, S.I., Hong, S.H. and Hong, S.H., 2006, "Microstructures and tensile behavior of carbon nanotube reinforced Cu matrix nanocomposites", Materials Science and Engineering: $A$, vol. 430, no. 1-2, pp. 27 to 33.

19. Kim, K.T., Cha, S.I. and Hong, S.H., 2007, "Hardness and wear resistance of carbon nanotube reinforced Cu matrix nanocomposites", Materials Science and Engineering: A, vol. 449-451, pp. 46 to 50.

20. Rajule, N., 2014, Ph.D. Thesis: Laser Synthesis and Characterization of Copper-Single Walled Carbon Nanotubes Nanocomposites, Department of Mechanical and Aerospace Engineering, North Carolina State University, Raleigh, NC, USA.

21. Tu, J.F., Rajule, R., Molian, P., and Liu, Y., to appear, "Laser synthesis of a copper-single-walled carbon nanotube nanocomposite via molecular-level mixing and non-equilibrium solidification," Journal of Physics, D: Applied Physics, 11 pages. 
22. Colomer, J.-F. , Henrard, L. , Lambin, Ph., and Van Tendeloo, G., 2002, "Electron diffraction and microscopy of single-wall carbon nanotube bundles produced by different methods," The European Physical Journal B, vol 27, pp. 111-118, DOI: 10.1140/epjb/e20020135.

23. Qin L-C, 2006, "Electron Diffraction from Carbon Nanotubes,", Rep. Prog. Phys. 69 (2006) 2761-2821, doi:10.1088/0034-4885/69/10/R02

24. Folmer, J. C. W., Withers, R. L., Welberry, Martin, J. D., 2008, "Coupled orientational and displacive degrees of freedom in the high temperature plastic phase of carbon tetrabromide, $\alpha-\mathrm{CBr}_{4}{ }^{\prime \prime}$, Phys. Rev. B., vol 77, pp. 144205:1-10. 

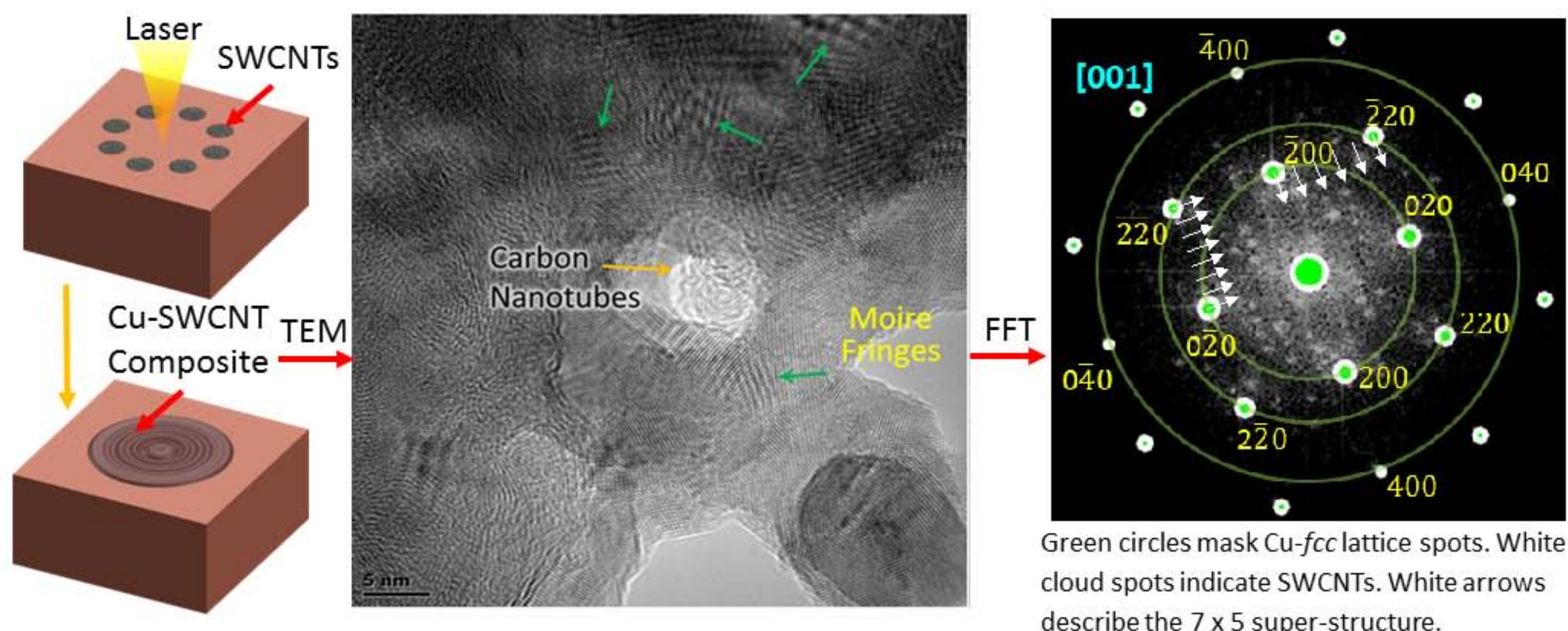

Green circles mask Cu-fcc lattice spots. White cloud spots indicate SWCNTs. White arrows describe the $7 \times 5$ super-structure. 